\title{
Índice de nombres de la Guía del Archivo de la Antigua Academia de San Carlos 1781-1800
}


INDICE DE NOMBRES DE LA GUIA DEL ARCHIVO DE LA ANTIGUA ACADEMIA DE SAN CARLOS

$1781-1800$ 


\section{Índice de nombres de la \\ Guia del Archivo de la Antigua Academia de San Carlos}

$1781-1800$

Formado por

Danilo Ongay Muza

SUPLEMENTO 2 DEL NÚM. 40 DE LOS

ANALES DEL INSTITUTO DE INVESTIGACIONES ESTÉTICAS

MEXICO, 1971 
Primera edicion: 1971

DR (C) 1971, Universidad Nacional Autónoma de México Ciudad Universitaria. México 20, D. F.

Dirección General de Publicactones Impreso y hecho en México 
La Guía del Archivo de la Antigua Academia de San Carlos. 17811800 , por Justino Fernández, se publicó como suplemento 3 del número 37 de los Anales del Instituto de Investigaciones Esstéticas, México (UNAM), 1968. Ahora se publica el indice de nombres de dicha Guía, formado por Danilo Ongay Muza. 


\section{Índice de nombres}

\section{$A$}

Abilez, Fernando 19

Acuña, Pedro de 89

Acuña, Ramón 15

Acuña y Troncoso, Cosme de 27, 29, 30, 31, 34, 36, 38, 40, 42, $43,44,45,48,56,57,60,64,67,68,69,75,76,77,89,98$, 111,113

Águila, Mariano del 22, 54, 94

Aguirre, Francisco 56, 71

Aguirre, José Ángel de 33, 70

Aguirre, Manuel José de 87

Aguirre, Mariano José de 47

Aguirre, Tomás de 23

Aguirre y Abendaño, 28

Álava, Manuel de 95

Albear, Miguel 44

Alcántara de Quintana, Pedro 18

Alcíbar, José de 12, 20, 45, 46, 55, 57, 70, 76, 77

Alemán, Juan Vicente 25

Alemán, Theodoro 17

Alessandri, José 39

Alfaro, José María 71, 89

Álvarez, Diego 46, 85

Álvarez, José 65, 70

Amán, Rafael 16

Amat de Tortosa, Andrés 79

Amezcua, Juan de 15 
Amusquiban, Francisco de 24

Andrade y Herrera, Ildefonso de 19

Ángeles, José Narciso de los 92

Antonio, Fray Obispo de Michoacán, Valladolid 15

Antúnez, José 23

Apartado, Marqués del 62

Aranda, Manuel de 106

Arce y Echegaray, José María 106

Aredo, Miguel Calixto de 20

Arias, Francisca 41, 81

Arias, José 29, 81, 86

Arias, Manuel 27, 35

Arias, Paula 4l, 81

Arias Centurión, José 40,41, 42, 59, 60

Arostegui, Antonio Clemente de 25

Arrospide, José María 78, 92, 94

Arssu y Arcaya, José Antonio de 19

Arzeo, José Severo 67

Asturias, Príncipe de 79

Audelo, Juan Marcelo 73, 74

Avila Roxano, José de 97, 108, 109

Axpec, Francisco de 72

Ayala Matamoros, José de 47, 50

Azanza, Miguel José de, Virrey 112, 114

Azoitia, Ignacio de 22

\section{$B$}

Bachiller y Mena, Rafael 103

Balderrama, José 71

Bargas, Nicolás de 68

Barragán, Theodoro Antonio 45

Barreda, José Ignacio 71

Barrio Junco y Esprisallo, F. de 16

Barrios, José María 103

Barroso y Torrubia, Antonio 11, 12, 28, 38, 41, 50, 52, 59, 60, $70,92,95$

Bartolache, José Ignacio 8, 12, 20, 21, 26 
Basoco, Antonio 28, 34, 38, 41, 50, 69, 70

Baxamar, Marqués de véase Porlier, Antonio

Bayer, Francisco 66, 113

Bayo, José Agustín 17

Belez de Escalante, Domingo 19

Berdugo, Pedro 20

Blas de Echendía, Leandro 16

Boamonde, Bernardo 24

Bobadilla, Sebastián 107

Bodega, Juan de la 82

Bonavia, Bernardo de 41, 47, 58, 59, 62, 63, 70

Bonilla, Antonio 33, 35, 38, 41, 52, 53, 56, 59, 60, 62

Branciforte, Virrey de 86, 96, 97, 98, 100, 102, 103, 104, 107, $108,109,110,112$

Bravo, Francisco 71

Bruno, Juan Antonio 44

Bruno de Ureña, Francisco 65

Buitrón y Velasco, José 89, 99

Bustamante, Manuel 80

Bustamante, Mariano 44, 89

Butrón, José 65, 70

C. N., Ángel de 18

Callado Posada Platas, Nicolás 17

Calvo, Nicolás 29

Camarón, José 96

Canal, Mariano Loreto de la 25

Canaliz, Antonio Ramón 16

Cándamo, Gaspar 103

Cantos, Joseph de 15

Caplera, Francisco 20, 55, 56, 57, 61, 70, 71, 75, 76, 77, 113

Cárdenas, Antonio de 23

Carlos I 9

Carlos III 10

Carmona, José 111

Carrera y Aramburu, Antonio 91 
Carrillo Pimentel, Agustín 24

Carvajal, Ciriaco 85

Casa Flores, Conde de 79

Casarín, Joaquín 28

Casasola, José de 24

Cassador, Felipe 15

Castañeda, José Luciano 67

Castera, Ignacio 58, 59, 65, 70, 77, 88, 100

Castilla, José de 15

Castilla, Mariscal de 12

Castillo, Juan José Gunemundo del 43

Castillo y Echendía, Manuel del 16

Ceraín, Adrián de 18

Cerda, José Ignacio de la 30

Cerezo, Agustín 61

Cervantes, Antonio 20, 55

Ciria, Marqués de 12, 14, 28, 34, 38, 41, 50, 70, 90

Conique, José de 89

Constanso, Miguel de 20, 21, 30, 32, 33, 41, 47, 58, 59, 62, 63, $65,70,88,89,96,99,100,105,106$

Contador, Felipe 18

Contador, Rafael 18

Contramina, Conde de véase González de Castañeda, José

Contreras, 65

Cora, Juan Antonio de 25

Córdova, Fernando de 34, 37, 42, 44, 49

Cornejo, Manuel 19

Corral, Miguel del 18

Corvera, Juan de 17

Crespo, Francisco Antonio 12, 18, 28, 62

Crouset, Juan 88

Cruz, Felipe de la 92

Cruz, Juan de la 44, 45, 46, 77

Cruz, Manuel de la 46

Cruz, Miguel de la 46

Cruz Fortes, Juan de la 95

Cuadrado, Miguel 80

Cuevas y Aguirre Avendaño, José Ángel de 12, 24, 28, 34, 35, $37,47,50,52,58,97$ 


\section{$\mathrm{CH}$}

Chávez y Mendoza, Luis de 79

\section{D}

Dávila, Policarpo 16

Delgadillo, José Eligio 65

Delgado y Fuentes, José María 111, 112

Díaz de Castro, Juan 17

Díaz de la Vega, Silvestre 105

Díaz Escandón, Pedro 17

Díaz González, Juan 23

Díaz y Alcántara, José 18

Díaz y Calixto Sans, Rafael 28

Diego, Francisco 73

Diez de la Mora, Miguel 73

Domínguez, Juan Francisco 45, 46

Duque, José María 67, 69

Durán de Velasco, Francisco 19

Durrey, Juan 91

\section{$E$}

Echegaray, Tomás de 75

Elhuyar, Fausto de 52, 62, 63, 70, 96

Escobar, José Esteban de 24

Escovedo, Francisco Alonso de 15

Espinosa de los Monteros, Mariano 22

Esquivel, Joaquín 71,113

Esteve, José 13, 21, 30, 38

Estrada, Antonio Teodoro de 108

Estrada, Ignacio 71

Eznal, Diego 23

\section{$F$}

Fabregat, José Joaquín $31,39,49,53,54,55,60,68,70,75$, $77,84,92,94,95,97,103,113$ 
González de Cossío, Francisco 20

González de Mesa, José 15

González de la Vega, Francisco 105

González Gómez Maldonado, Luis 51

González Guiral, Manuel 49

González Montañez, Juan Antonio 86

González Velásquez, Antonio 27, 28, 29, 34, 38, 47, 48, 49, 50, $51,58,59,60,65,66,67,69,70,75,77,78,80,82,83,84$, $89,97,98,99,101,104,105,106,107,108$

Gordillo, Francisco 96

Gorospe, Rafael María de 19

Gorráez, Joseph de 11

Gorrindo Palomino, Pedro 18

Goya y Lucientes, Francisco 66

Goycoechea, Ignacio 64

Granados, Mariano 88

Guadalajara y Tello, Diego de 52, 61, 66, 67, 70, 76, 77, 84, 87, 88, 92, 93, 95, 97, 99, 102, 103, 104, 108, 112

Guerrero, Donaciano 109

Guerrero, Francisco 65

Guerrero, José María 70, 75, 76, 85, 87, 91, 95, 99, 101, 113

Guerrero, Juan Ignacio 22

Guerrero, Mariano 71, 113

Guerrero y Torres, Francisco Antonio 65, 70, 75, 77, 78, 80, 85

Guridi y Alcocer, José 113

Gutiérrez, José 35, 77, 79, 83, 91, 95, 96, 98, 99, 100, 101, 105, $106,111,113$

Gutiérrez, Luis 28

Gutiérrez N. de 34

Gutiérrez, Rafael Joaquín de 20, 55, 65, 70, 71, 76, 77, 113

Gutiérrez de Castañeda, Francisco 19

Gutiérrez de los Ríos, Joaquín 92

Gutiérrez y Heredia, José 66

\section{$H$}

Heredia, José Joaquín de 64, 66, 78, 91, 93, 100, 104

Hernández Chico, José 25

Hernández Pareja, Eusebio 15 
Herrera, José Mariano de 97

Herrera, Vicente de 20

Horge, Antonio Lorenzo de 25

Huerta, José 75, 92

Hurtado, Juan 71

Ibarra, José 65

Ibarrola, Tomás Ramón 23

Icauna, Manuel Antonio Fernando 23

Iñaqui, Rafael 34

Irigoyen, Miguel Francisco de 19

Iturbide, Pedro Antonio de 19

Iturrigaray, Virrey 83

$J$

Jarero, Pedro 17

Jáuregui Brunes, Pedro 24

Jiménez Caro, Ildefonso 55

\section{$L$}

Labarrieta, Domingo 22

Labastida, José de 113

Ladrón de Guevara, Baltazar 83, 84, 107, 108

Ladrón de Guevara, Joaquín José 109

Lagoa y de Miranda, Domingo 23

Larraguita, Francisco 17

Larrañaga, Bruno 102

Larrañaga, José Rafael de 92

Larrañaga, Norberto Francisco 12

Lassaga, Diego de 18

Lassaga, Juan Lucas de 11, 12, 95

Leal, Francisco D. 15

León, José Mariano de 91

León, Lucas 71 
León Caballero y Ontiveros, Mateo de 108

Lerena, Pedro de 81

Luezo, Manuel Nicolás de 16

Liceaga, Ramón Luis de 11, 52, 62

Lilia, Francisco 24

Lindo, Francisco 43, 69, 99

Lissa, Francisco de 19

Lizardi, Miguel de 23

Loazes, Fernando 22

Longinos Martínez, José 40, 42

López, Andrés 20, 34, 55, 70, 113

López, Anselmo 28

López, José 73, 77

López, Juan 77

López, Manuel José 44, 45, 46, 69, 87, 89, 91, 99, 101

López, Nicolás 73

López de Pareja, Juan 72

López de Sagredo, Juan Ramón 16

López Dena, Dionisio 113

López Marchena, Julián 99

López Portilla, Nicolás 17

Loreto Villanueva, José 75

Lovis, Agustín 67

Luelmo, Francisco 55

Lugo, Ángel 15

Luis, Fray. Obispo de Yucatán, Mérida 15

Luyando, Ruperto Vicente de 20

\section{$L L$}

Llaguno, Eugenio de 98, 110

Llano, José Mariano de 24

Llano y Sierra, Francisco Antonio del 19

Llanos, José Mariano de 19

\section{M}

Maella, Mariano 65, 91

Maín, José 71 
Malaspina, 8I

Maldonado, Pedro 72

Mandicutt, Lorenzo A. 24

Mangino, José Fernando $7,8,11,12,20,21,22,28,30,32$, $35,37,38,50,57$

Mangino Román de Posada, Fernando 28, 66

Mansi, Domingo 113

Manzano de Almanza, José 23

Mar López, Pedro 64

Marañón, José 25

Marchena, Juan 22, 54

Marchena, Julián 94

Marenco, José Luis 22, 66, 67

Mariano, Fray 92

Marín, Gerónimo 72

Mariscal, Francisco 22

Mariscal de Castilla véase Ciria, Marqués de

Marqués del Apartado 67

Márquez de Ochoa, Pedro José 31

Marrugat, José 75

Martín Alonso, Luis de 35, 44, 64, 67, 70, 75, 111

Martínez, Francisco 60

Martínez, Luis 44, 61

Martínez Moreno, José 16, 22

Martínez Sanz de Olmedo, José 113

Mateo Valenzuela, José 75

Mateo y Romeo, Alejandro 18

Maurino, Juan José 22

Mayorga, Martín de 7, 11, 12

Mazo Avilés, José del 63, 64, 65, 70, 85, 100, 102

Medina, Francisco A. de 14

Medina y Torres, Conde de 14

Melgar, Estevan 16

Méndez Prieto, Antonio 51

Mendiola, Tomás Francisco 24

Mendívil, Andrés 103

Mendoza, Andrés de 73

Mendoza, José Mariano 97

Menocal, Ángel de 64 
Mestre, Manuel 103

Mestre y Pardo, Diego 22, 32, 39, 49, 51, 53, 55, 61, 62, 64, $69,70,93$

Michaus, Martín Ángel de 23

Mier y Trespalacios, Cosme de 103, 107, 108, 111

Miguel Ángel 71

Miguel de Jesús, Fray 41

Miranda, Miguel Ignacio de 13

Molina, Antonio de 18

Molina y Garrido, José 29

Moncada y Berrio, María Guadalupe de 96

Montero, José 113

Montero y Austri 16, 25

Montero y Velarde, Antonio 79

Montes, José Antonio 66, 67

Montes de Oca, José María 44, 45, 69, 78, 99, 101

Montoya, Lorenzo 18

Mora y Peysal, Antonio de 103

Morales, Antonio Calixto 28

Morales, José Antonio de 12, 28

Morelos, Mariano 113

Moreno, Manuel 61, 62

Moreno, Pedro 23

Moreno Texada, José 98, 102

Mosti, Antonio José 93

Mungarrieta, Juan Felipe 25

Muñiz, Narcizo 17

Muñoz, Ignacio 14

Muñoz, José Marcelo 113

Muñoz, Manuel 44, 45, 67, 88

Muñoz, Mariano 32

Muñoz de Pisón, Juan Antonio 25

\section{$N$}

Nadal y Zubet, Jacinto 74

Nápoles, Cristóbal 75

Navarro, Juan 24 
Noreau de Sisson, Pedro Fermín 16

Noriega, Bruno María de 105

Núñez de Haro, Alonso 14, 86

\section{$O$}

Obispo de Puebla 19

Obregón, Ignacio de 18

Ocampo, Salvador de 73, 74

Onofre 71

Orcasitas, Manuel 32

Oriñuela, José 86, 87

Ortega, Pablo de 16

Ortega Montenegro, Bartolomé de 28

Ortiz, Domingo 113

Ortiz, Francisco 112

Ortiz, José Martín 64, 65, 70

Ortiz, Manuel 82

Ortiz, Sebastián 24

Ortiz de Castro, Francisco 91, 96

Ortiz de Castro, José Damián 20, 21, 29, 30, 32, 33, 47, 48, $65,70,77,86$

Ovando 40

\section{$P$}

Pablo, Fray 23

Paez de la Cadena, Miguel 17, 21

Pagola, Antonio de 15

Palacios, Francisco 40,64

Palomino, Antonio 81

Panes, Diego 18

Pardo, José Anastasio de 61, 62

Paredes, Conde de 9

Pasalle, Felipe Francisco H. 40

Paso y Troncoso, Pedro del 23, 24

Patiño Ixtolinque, Pedro Antonio 44, 45, 77, 78, 87, 91, 99 
Paz y Pinzón, Aptoval de 16

Peña y Flores, Francisco Antonio de la 42

Pérez, Francisco 32

Pérez, Gregorio 81

Pérez, Pascual 45

Pérez, Sebastián 14

Pérez Calasna, José 16

Pérez de Ávila, José Manuel 67

Pérez de Herrera, F. 24

Pérez de Solís, Manuel 112

Pérez de Zadaire, Pedro 25

Pérez Marañón, Fernando 103

Pérez Toledano, Manuel 16

Pérez Valdés, Diego 97, 111

Pesa y Casas, Ignacio José de la 18

Picaso, José Mariano 90

Pineda, Antonio 79

Pinzón, Juan Antonio 24

Piñeiro, Antonio 9, 13, 21, 22, 23, 24, 25, 26, 28, 31, 32, 35, 42, 48, $51,52,54,56,59,60,70,80,84,107$

Piso, Francisco 90

Polanco, José Antonio 71, 79

Polanco, Valentín 71

Porlier, José Antonio 31, 36, 38, 40, 42, 49, 51, 52, 54, 59, 60, 62, $68,79,81$

Posa, Francisco Benito 32

Posada, Ramón de 8, 9, 10, 32, 34, 36, 37, 39, 41, 42, 43, 47, 48, $50,51,52,53,56,57,58,59,61,62,70,75,79,80,95,98$

Prieto, Lucas 73

Frieto de Bonilla, Ildefonso José 51

Pulgar, José María 80, 99, ].02, 109

\section{$Q$}

Quevedo, Pedro de 17

Quintana, Aimes Mariano de 18

Quintana, Francisco Juan 24

Quintano, Pedro Miguel 17 


\section{$R$}

Rafael Joseph, Fray. Obispo de León. Ciudad de Monterrey 15

Ramírez, Manuel 19

Ramos, Francisco Xavier 110, 111, 113

Razo, José del 77

Reales, Francisco 13, 30

Retama y Martínez, Mariano Isidoro 103, 109

Revillagigedo, Virrey Conde de 36, 41, 54, 57, 60, 62, 63, 68, 70, $76,79,80,81,84,85,87,89,94,97,98,99$

Reyes, Francisco 59

Reyes, Manuel 113

Reyes, Mariano Pantaleón 65

Keyes y Torres, Ignacio 111

Reynoso, Manuel 71

Riaño, Juan Antonio 103

Riaza y Velasco, Bernabé de 17

Rica, Luis 16

Ricarte y Espinosa 54

Ríos, José Vicente de los 25

Rivas Cacho, Marqués de 14

Rivera, Miguel de 25

Rivera, Nicolás Francisco de 19

Roa, Andrés de 72

Robles, Nicolás de 25

Rodallega, José María 109

Rodríguez, José Joaquín 13, 30

Rodríguez, Pedro 88

Rodríguez Alconedo, José 96

Rodríguez de León, Juan 113

Rodríguez de Velasco, Antonio 51, 95

Rodríguez Juárez, Juan 65

Rojas, Juan de 73, 74

Fomano, Felipe 28

Romero, José Alonso 24

Romero, Juan José 113

Romero, Paz 19

Romero de Caamaño, Joaquín 47, 50, 62, 102

Rosa, Ignacio de la 46 
Ruiz de Cabañas, Juan Cruz. Obispo de Guadalajara 106

Ruiz de Reyna, Manuel 39

Ruscerio, Matías 24

\section{$S$}

S., Lorenzo 25

Sabarrieta, Domingo de 16

Saenz, Juan de 20, 34, 55, 70, 71

Sagas, Andrés 25

Salbago, Diego Antonio 82

Salcedo, Antonio de 72

Salcedo, José Cleto Nepomuceno 28, 29

Sales Carrillo, Francisco de 28

Samaniego y Enderica, Saturnino 101, 108

San Jorge, Marqués de 106, 108

San Miguel de Aguayo, Marqués de 12, 28, 34, 38, 41, 50, 70

San Romás, Marqués de 83

Sancha, Gabriel de 94

Sánchez, Ignacio 104

Sánchez, Juan 77, 95

Sánchez Bernal, Gerónimo 28

Sánchez Carbajal, Rafael 18

Sánchez de Cañas, Florentino 17

Sánchez de Pareja, Francisco Xavier 19

Sánchez de Tagle, Manuel Estevan 19

Sánchez de Torres, José Mariano 24

Sánchez Solache, Ildefonso María 16

Sánchez y Torres, Martín Antonio 24

Sánchez Vallesteros, Francisco 19

Sandoval, Bartolomé 103

Sandoval, Ignacio 35, 77, 85, 87, 91, 92

Sandoval, Juan Mariano 35

Sandoval, Santiago Cristóbal 20, 45, 46, 55, 70, 77

Sandoval y Austria, Rafael Tiburcio 45

Santa María Inchaurregui, Antonio de 75, 104

Santelicer, José María 93

Santelicer Pablo, Juan Eugenio 93

Santiago, Conde de 62 
Santo, Blas Mariano de 22

Saravia, José Mariano 13, 30

Sarmiento, Julio Simón 24

Selma, Fernando 27

Septién, Pedro Antonio de 16, 25

Seré, Martín 84

Serna, José de la 15

Serna, Manuel de la 20,34, 55, 70

Serralde, Juan lgnacio 113

Silva y Ahoguera, Juan de 18

Sonora, Marqués de 26, 27, 28, 29

Sonora, Marquesa de 30

Soto, José 28

Soto Mayor, Raimundo 15

Suárez, Diego 16

Suárez, Vicente 82

Suelmo, Francisco 22

Suría, Tomás 13, 30, 35, 38, 81, 84

\section{$T$}

Tapiz, Pedro Manuel 78

Tejedor, Eusebio 66

Tello de Orozco, Francisco Xavier 105

Tello y Somosa, Manuel de 18

Teruel, Felipe Antonio 95

Tirado, Mariano 68

Tola y Salcedo, Luis de 67, 84, 97, 104, 109, 111, 113

Tolsá, Manuel 29, 64, 68, 70, 76, 77, 80, 81, 83, 85, 86, 88, 91, 95, $96,97,100,101,102,103,104,106,107,113$

Torre, Ignacio de la 110

Torres, Francisco 84

Torres, José Joaquín de 50, 100

Torres, Nicolás de 73

Torres y Daza, Miguel de 17

Trelles, José 39, 49

Troncoso, Gerónimo José 58 


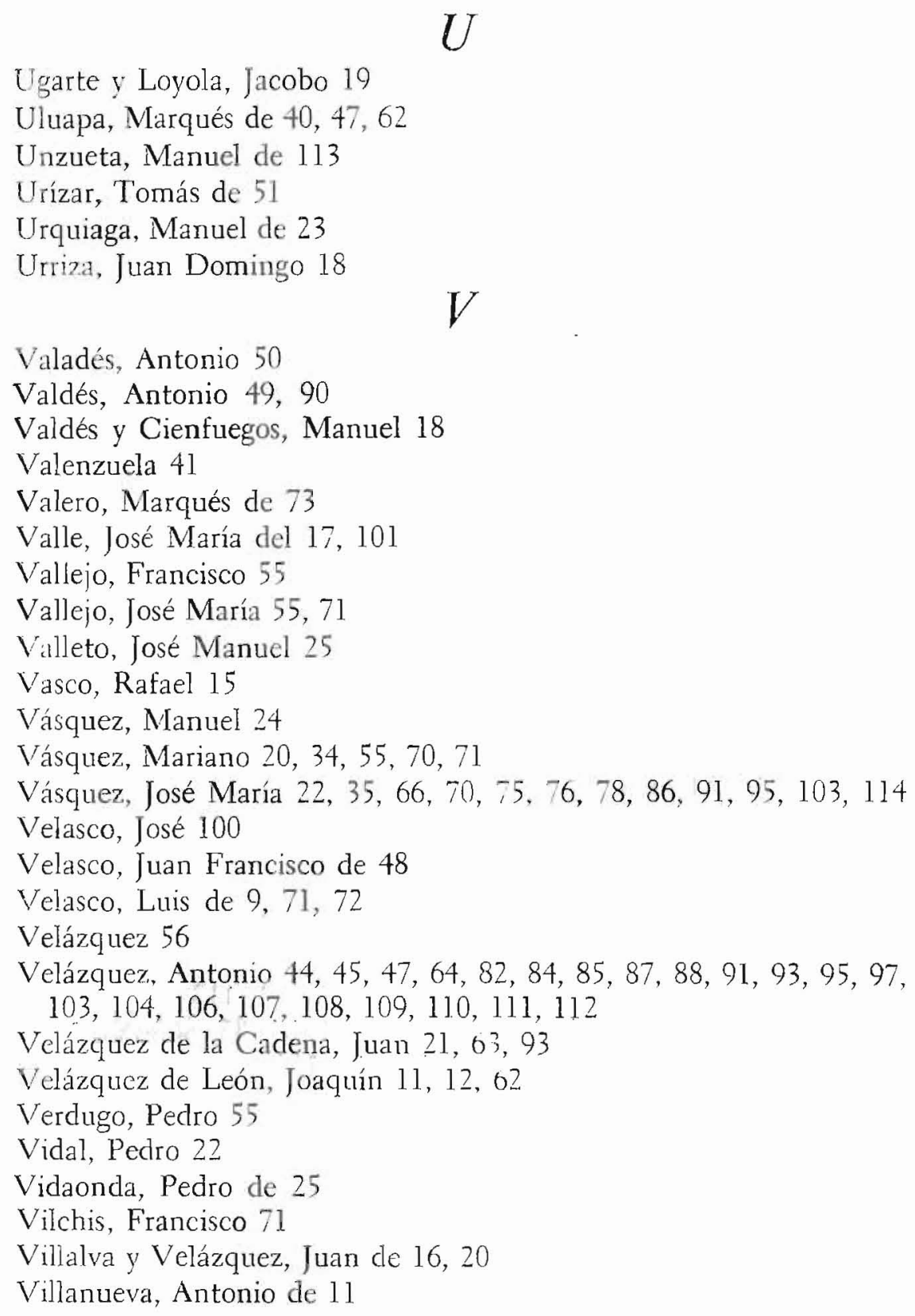


Villanueva, José Loreto 107

Villar del Águila, Marqués de 14

Villarreal, José Francisco 40, 42

Villasante, Diego 24

Villerías, Miguel 78

\section{$X$}

Ximénez, Buenaventura 17

Ximénez Bohorques, Joaquín 24

Ximénez Caro, Alonso 20

Ximénez de Ocón, Pedro 22

Ximénez de la Plaza, Pablo 11

Ximénez Ferenero, Juan Antonio 17

Ximénez y Zevallos, José 24

Ximeno y Planes, Rafael 93, 97, 98, 101, 113

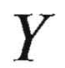

Yáñez, Agustín 15

Yermo, Antonio 25

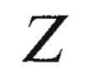

Zamarripa, José María 36, 43, 75

Zárate, A. de 16

Zaubazaín, José Mariano 64

Zedillo, Joaquín Zeferino 15

Zepeda, Mariano de 74

Zerezo, Agustín 22

Ziprían, Juan Antonio de S. 18

Zubinez, Mariano Félix de 17 
En la imprenta Universitaria, bajo la direccion de Jorge Gurría Lacroix, se terminó la impresión de fndice de nombres de la guía del archivo de la antigua academia de San Carlos, 1781 1800, el día 18 de octubre de 1971. Su composición se hizo en tipos Electra 11:12 y 8:9. La edición consta de 1500 ejemplares. 
